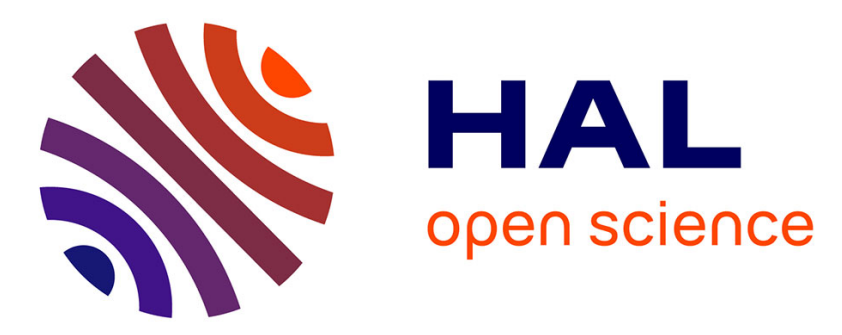

\title{
Ensemble expérimental de mesures optiques sous irradiation électronique
}

E. Mercier, G. Guillot, A. Nouailhat

\section{To cite this version:}

E. Mercier, G. Guillot, A. Nouailhat. Ensemble expérimental de mesures optiques sous irradiation électronique. Revue de Physique Appliquée, 1977, 12 (1), pp.61-65. 10.1051/rphysap:0197700120106100 . jpa-00244120

\section{HAL Id: jpa-00244120 https://hal.science/jpa-00244120}

Submitted on 1 Jan 1977

HAL is a multi-disciplinary open access archive for the deposit and dissemination of scientific research documents, whether they are published or not. The documents may come from teaching and research institutions in France or abroad, or from public or private research centers.
L'archive ouverte pluridisciplinaire HAL, est destinée au dépôt et à la diffusion de documents scientifiques de niveau recherche, publiés ou non, émanant des établissements d'enseignement et de recherche français ou étrangers, des laboratoires publics ou privés. 
Classification

Physics Abstracts

$0.640-7.183-8.846$

\title{
ENSEMBLE EXPÉRIMENTAL DE MESURES OPTIQUES SOUS IRRADIATION ÉLECTRONIQUE
}

\author{
E. MERCIER, G. GUILLOT et A. NOUAILHAT \\ Laboratoire de Physique de la Matière $\left(^{*}\right)$ \\ Institut National des Sciences Appliquées de Lyon \\ 20, avenue Albert-Einstein, 69621 Villeurbanne Cedex, France \\ (Reçu le 4 mai 1976, révisé le 16 juillet 1976, accepté le 3 août 1976)
}

\begin{abstract}
Résumé. - On décrit un appareillage permettant de mesurer simultanément plusieurs paramètres optiques d'un cristal soumis à une irradiation électronique continue ou pulsée, en particulier l'absorption optique (spectres ou cinétiques) et la luminescence (spectres, cinétiques et temps de déclin). On donne des exemples concernant l'étude de l'extinction de la luminescence par les défauts créés par irradiation dans les halogénures alcalins.
\end{abstract}

\begin{abstract}
We describe an apparatus which allows the simultaneous measurement of several optical parameters, especially the optical absorption (spectra or kinetics) and the luminescence (spectra, kinetics and lifetimes) of a crystal irradiated by a pulsed or continuous electron beam. A few examples related to the quenching of the luminescence of alkali halides by the defects created by irradiation are given.
\end{abstract}

1. Introduction. - Dans le cadre d'une étude concernant les processus induits par irradiation dans les solides ioniques, différents paramètres associés au cristal doivent être mesurés simultanément : nature et concentration des centres formés, évolution de ceux-ci en fonction de la dose dans une large gamme de concentrations, de même que des caractéristiques des luminescences intrinsèques ou extrinsèques (cinétique, spectre, temps de déclin).

Nous avons réalisé un appareillage d'encombrement réduit et de conception modulaire suffisamment simple pour s'adapter facilement à des conditions d'expériences différentes. L'irradiation électronique a été choisie pour avoir, en particulier, une forte concentration en défauts mesurable par absorption optique dans un temps relativement limité (de l'ordre de $10^{3} \mathrm{~s}$ ). Les phénomènes étudiés étant généralement fonction de la température, celle-ci est régulée au niveau du porteobjet pour travailler dans des conditions fixes ou variables entre $4 \mathrm{~K}$ et $300 \mathrm{~K}$. L'absorption optique est mesurée par un spectromètre à double ou simple faisceau qui permet d'effectuer des spectres dans le domaine 200-1 $000 \mathrm{~nm}$ même dans les zones spectrales où le cristal est fortement luminescent. En mesures cinétiques, on peut enregistrer simultanément les variations de concentration de deux centres différents dans un même cristal sous irradiation. Ceci permet une plus

$\left(^{*}\right)$ Equipe de recherche associée au C. N. R.S.

REVUE DE PHYSIQUe APPLIQUÉE. - T. 12, No 1, JANVIER 1977 grande précision en éliminant l'erreur possible due aux fluctuations statistiques entre cristaux. Les sources d'excitation et les dispositifs d'analyse de la luminescence complètent l'ensemble instrumental. Le faisceau électronique est pulsé pour les mesures des temps de déclin et de cathodoluminescence faible (détection par boxcar et amplificateur synchrone). Ce dispositif se distingue essentiellement de celui présenté par Levy et al. [1] par sa plus grande souplesse d'emploi (domaine de température beaucoup plus large, grande possibilité de variation du taux de dose) et par le fait qu'il permet de combiner des excitations ultraviolettes et électroniques et de mesurer des durées de vie de luminescence.

2. Description de l'appareillage. - L'enceinte d'expérimentation d'un volume de 6 litres, sur laquelle se trouvent le cryostat et le dispositif d'irradiation électronique, est montée sur un bloc de pompage permettant l'obtention d'un vide propre de $10^{-7}$ torr environ nécessaire au bon fonctionnement du canon à électrons. Pour éviter la pollution de la surface de l'échantillon au cours du temps, particulièrement critique dans les mesures d'absorption ou de stimulation dans l'ultraviolet, des précautions importantes ont été prises : (i) un piège à zéolithe évite la rétrodiffusion d'huile de la pompe primaire, (ii) la pompe secondaire, type bloc intégré Edwards $63 / 150 \mathrm{M}$, est pratiquement sans rétrodiffusion et des écrans refroidis dans l'enceinte d'expérimentation complètent la protection de l'échantillon 
L'absence de toute pollution au niveau de l'objet a été testée, dans les conditions expérimentales de température, par des mesures d'absorption optique de longue durée dans le domaine d'absorption de l'huile utilisée (Santovac 5).

2.1 Cryostat. - On dispose de deux cryostats régulés en température, l'un à azote liquide construit au laboratoire, et l'autre à circulation d'hélium fabriqué par Thor Cryogenics qui permet des mesures jusqu'à $4 \mathrm{~K}$ avec une stabilité de $\pm 0,25 \mathrm{~K}$. La température du porte-objet du cryostat à hélium est mesurée par un thermocouple AuFe 0,03 at. \%-chromel avec référence à l'azote liquide, celle du cryostat à azote est mesurée par une sonde de platine. L'étalonnage de la température de l'objet au voisinage de l'hélium liquide est fait en utilisant les propriétés des luminescences intrinsèques de CsI entre $4 \mathrm{~K}$ et $12 \mathrm{~K}$ [2]. Ceci permet de vérifier la qualité du contact thermique entre l'échantillon et son support obtenu par pression sur l'objet d'une lame à ressort. Chaque porte-objet comporte deux trous permettant de positionner deux cristaux dans des conditions équivalentes pour des mesures d'absorption optique en double faisceau.

2.2 SYSTÈME D'IRRADIATION ÉlECTRONIQUE. - La figure 1 montre la disposition du système d'irradiation

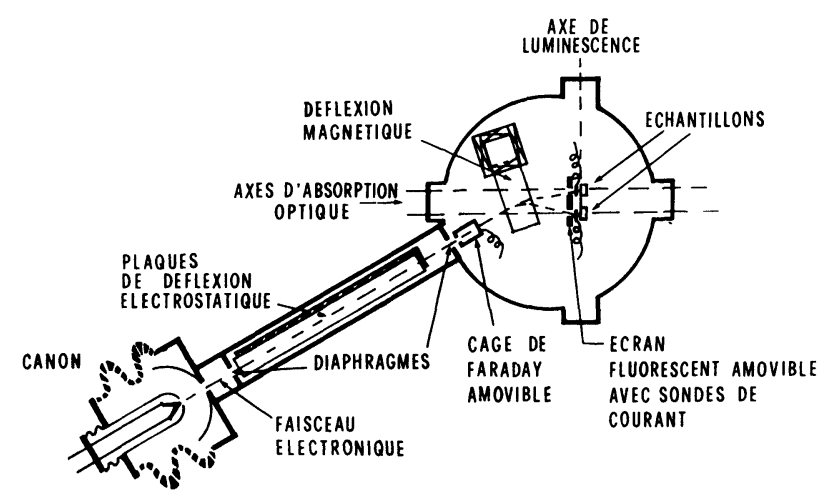

FIG. 1. - Dispositif d'irradiation électronique.

électronique. La haute tension est fournie par une alimentation Brandenburg $907 \mathrm{~N}(-6 \mathrm{kV}$ à $-60 \mathrm{kV})$ stabilisée à $0,01 \%$, protégée en débit à $400 \mu \mathrm{A}$. Le canon triode, construit au laboratoire, fournit, au niveau des échantillons, un faisceau large dont on a prélevé la partie centrale par un diaphragme et dont l'homogénéité en densité de courant est de $\pm 2 \%$. Le canon, extrêmement stable en régime de charge d'espace, permet un réglage de débit dans une large gamme : $0,1-10 \mu \mathrm{A} / \mathrm{cm}^{2}$ en continu. Ces conditions sont parfaitement adaptées aux mesures sur les halogénures alcalins. Par exemple, pour $\mathrm{KBr}$ où la profondeur de pénétration des électrons est donnée par la formule suivante [3] :

$$
d=0,035 \mathrm{~V}^{1,7} \quad \text { avec } d \text { en micron, } V \text { en kilovolt }
$$

le taux de déposition d'énergie en $\mathrm{eV} / \mathrm{cm}^{3}$.s varie entre $10^{19}$ et $5 \times 10^{21}$. Compte tenu de l'épaisseur de la couche irradiée, de l'énergie nécessaire à la formation des défauts et des phénomènes qui régissent l'accumulation de ceux-ci, nous pouvons mesurer, par absorption optique, des concentrations de défauts allant de $10^{16} / \mathrm{cm}^{3}$ à $5 \times 10^{19} / \mathrm{cm}^{3}$ pour des durées d'irradiations de l'ordre de $10^{3} \mathrm{~s}$.

Le canon électronique est relié à l'enceinte par un tube en acier inoxydable qui contient un dispositif de coupure rapide du faisceau par déflexion électrostatique (temps de coupure limité actuellement à $0,2 \mu \mathrm{s})$. La fréquence des pulses est donnée soit par le réseau $(50 \mathrm{~Hz})$, soit par un oscillateur $\left(10 \mathrm{~Hz}-10^{4} \mathrm{~Hz}\right)$ et leur largeur peut être comprise entre $50 \%$ et $0,1 \%$ de la période. Ce système permet, d'une part, une détection synchrone des faibles niveaux de cathodoluminescence, et, d'autre part, des mesures de temps de déclin de luminescence lorsque ceux-ci sont supérieurs à $0,5 \mu \mathrm{s}$. Le système de déflexion magnétique à l'intérieur de l'enceinte envoie le faisceau d'électrons sur l'un ou l'autre des échantillons sous une incidence quasi normale. Les axes optiques sont ainsi laissés libres pour les mesures d'absorption optique ou de luminescence.

La densité de courant du faisceau est mesurée avec une cage de Faraday amovible placée avant la déflexion magnétique et reliée à un microampèremètre. Le positionnement précis du faisceau et le contrôle de son homogénéité sont effectués au moyen d'un écran fluorescent mobile placé juste devant les échantillons et sur lequel sont disposées deux petites sondes de courant, chacune correspondant au centre d'un des deux cristaux.

2.3 SYSTÈME DE MESURES OPTIQUES. - Il est situé autour de l'enceinte d'expérimentation.

La figure 2 montre la disposition générale du système de mesures optiques. La source de lumière $S_{1}$ est utilisée pour le fonctionnement en spectromètre à double faisceau. En se servant des deux sources de

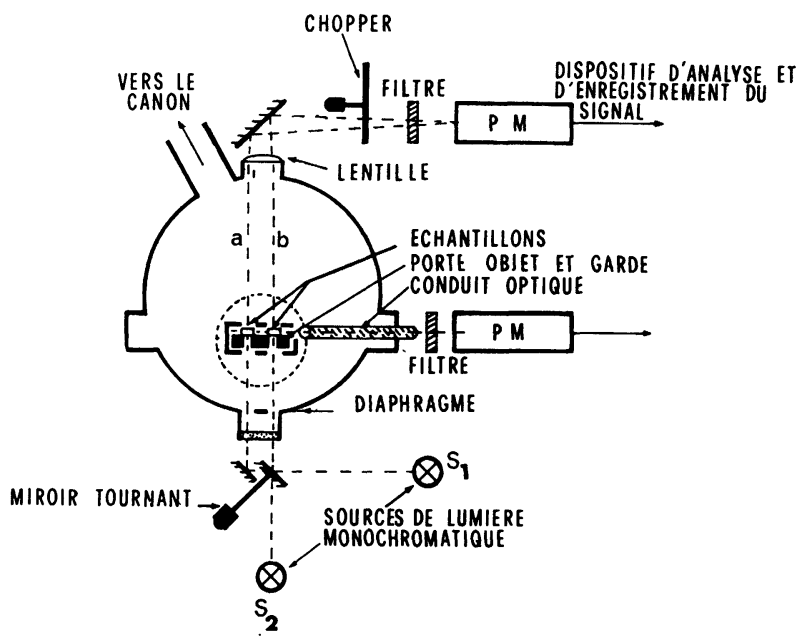

FIG. 2. - Dispositif du système de mesures optiques. 
lumière $S_{1}$ et $S_{2}$, et en obturant le faisceau a, il est possible de mesurer simultanément les cinétiques de formation des défauts correspondant aux longueurs d'onde de $S_{1}$ et $S_{2}$ dans l'échantillon de la voie b. La cathodoluminescence est recueillie par un conduit optique de quartz sur le côté des échantillons. Toutes les fenêtres de l'enceinte sont en quartz Tetrasil; le domaine spectral couvert par notre installation, compte tenu des récepteurs, s'étend de 200 à $1000 \mathrm{~nm}$. Le dispositif optique peut être modifié par rapport à la figure 2 et être adapté à l'étude de nombreuses propriétés optiques des cristaux polaires lorsque cellesci dépendent de la concentration en défauts : par exemple, le spectrophotomètre peut être remplacé par une source d'excitation ou de stimulation.

2.3.1 Mesures en spectrophotomètre à double faisceau. - Le spectrophotomètre à double faisceau comporte :

- deux sources de lumière : une lampe à filament de tungstène et une lampe à deutérium (Original Hanau D60 F) ;

- un monochromateur haute intensité Bausch et Lomb, avec réseaux UV, UV visible, IR, le tout constituant la source $S_{1}$.

A la sortie du monochromateur, un chopper lumineux qui sert en même temps de séparateur de faisceaux porte séquentiellement des secteurs identiques réfléchissants, absorbants et vides. Un miroir fixe, parallèle aux miroirs tournants, réfléchit le faisceau incident quand celui-ci passe à travers le chopper.

Les faisceaux $a$ et $b$ (Fig. 2) transmis par les cristaux sont collectés par une lentille et envoyés sur le photomultiplicateur de mesure (EMI 9529 B, RCA C7151 Z ou $150 \mathrm{CVP}$ suivant le domaine spectral couvert). Le signal électronique est amplifié, la partie correspondant aux secteurs absorbants du miroir tournant est asservi au niveau zéro, puis les signaux correspondant aux voies de mesure et de référence séparés par des portes commandant des mémoires analogiques. Le signal de mesure est divisé par celui de référence dans un diviseur analogique et le quotient envoyé sur un amplificateur logarithmique qui fournit ainsi directement la densité optique.

Ce dispositif présente l'avantage important de permettre une mesure de densité optique lorsqu'il y a dans l'enceinte de la lumière parasite continue, en particulier, une faible cathodoluminescence émise par le cristal.

\subsubsection{Mesures en simple faisceau sur deux longueurs} d'onde. - Dans les mesures simultanées sur deux longueurs d'onde, le faisceau à la deuxième longueur d'onde est détecté à la place du signal de référence ; on enregistre alors les mesures de transmission à la sortie des mémoires analogiques, et la densité optique est calculée par rapport à la transmission initiale.
2.3.3 Mesures d'absorption optique dans le cas de forts niveaux de luminescence. - Dans le cas où la cathodoluminescence est forte et où elle ne peut être supprimée par filtrage optique (en particulier pour des bandes d'absorption se recouvrant avec une bande d'émission), on place devant le photomultiplicateur un écran synchrone avec le miroir tournant qui obture la photocathode pendant la durée de l'irradiation qui est alors également échantillonnée de façon synchrone.

2.3.4 Mesures de luminescence. - Les mesures de luminescence se font indépendamment et simultanément aux mesures d'absorption optique. La lumière est captée à $90^{\circ}$ de l'axe d'absorption optique par une lentille ou par un barreau de quartz; l'analyse spectrale est faite par un jeu de filtres ou par un monochromateur. Suivant l'intensité du signal et le mode d'excitation de la luminescence, on travaille en continu ou en détection synchrone (emploi d'un amplificateur synchrone Brookdeal). L'excitation de la photoluminescence se fait selon les axes servant habituellement aux mesures d'absorption optique sur la face irradiée par une lampe au deutérium (Original Hanau D 200).

Un échantillonneur moyenneur boxcar (PAR 162) permet les mesures des temps de déclin.

3. Exemples d'application. - L'appareillage a été spécialement conçu pour l'étude des cinétiques de coloration sous bombardement électronique et de l'influence des défauts ponctuels sur diverses luminescences d'halogénures alcalins purs ou dopés. Lorsqu'un cristal est irradié à très basse température, il y a création d'excitons. Leur recombinaison radiative à partir des états relaxés singulet et triplet ${ }^{1} \Sigma_{\mathrm{u}}$ et ${ }^{3} \Sigma_{\mathrm{u}}$ est à l'origine des luminescences intrinsèques $\sigma$ et $\pi$ alors que leur recombinaison non radiative à partir d'un état inconnu conduit à la formation d'une paire de Frenkel : centre $F$ (lacune d'halogène ayant piégé un électron) et centre $\mathrm{H}$ (atome d'halogène interstitiel) [4].

Les trois exemples d'études que nous donnons illustrent principalement le fait que les caractéristiques de la luminescence excitonique (intensité et durée de vie) sont profondément modifiées par les défauts créés sous irradiation.

Le faisceau d'électrons est utilisé pour la formation de défauts et l'excitation de l'échantillon par création de trous et d'électrons libres. L'extinction de la photoluminescence du cristal pur est mesurée de la même façon car la profondeur de pénétration des électrons est supérieure à celle de la lumière de photoexcitation UV (de l'ordre de la longueur d'onde). En créant les défauts par irradiation électronique, nous avons alors l'avantage, sur les expériences où les défauts sont formés par irradiation UV [5], d'obtenir une coloration uniforme dans toute la couche irradiée et de pouvoir ainsi effectuer une mesure précise de la concentration en défauts.

Exemple 1: Cinétiques de formation des centres $\mathrm{H}_{\mathrm{A}}(\mathrm{Na})$ et d'extinction de la luminescence excitonique 
au voisinage des ions $\mathrm{Na}$ dans $\mathrm{KBr}$ faiblement dopé en $\mathrm{Na}$ à $80 \mathrm{~K}$.

Nous avons utilisé des cristaux de $\mathrm{KBr}$ de provenance Korth dont l'analyse a montré qu'ils contenaient environ $50 \mathrm{ppm}$ de $\mathrm{Na}$. Il est connu qu'à basse température le sodium joue le rôle de piège à interstitiel saturable et que, par irradiation, il se forme une bande d'absorption due au centre $\mathrm{H}_{A}(\mathrm{Na})$ (interstitiel neutre ou centre $H$ stabilisé au voisinage du sodium) à $410 \mathrm{~nm}$ [6]. L'exciton auto-piégé près du sodium est responsable d'une bande d'émission à $430 \mathrm{~nm}$ [7].

En effectuant simultanément des cinétiques respectivement en absorption et en émission à ces deux longueurs d'onde, nous avons montré que celles-ci étaient de forme exponentielle et de même constante de temps. Nous avons ainsi mis directement en évidence le fait que l'interstitiel agit comme centre extincteur sur les centres luminescents [8].

Exemple 2: Variation de la constante de temps de la luminescence excitonique $\pi$ dans $\mathrm{KBr}$ à $10 \mathrm{~K}$ avec la concentration en défauts.

Pour l'enregistrement des temps de déclin, le canon est utilisé en régime pulsé. La concentration en centres F (lacune d'halogène ayant piégé un électron) est mesurée simultanément par absorption optique à $601 \mathrm{~nm}$. A $10 \mathrm{~K}$, le déclin pour le cristal vierge est exponentiel et de constante de temps $\tau_{\mathfrak{a}}=100 \mu \mathrm{s}$, valeur en accord avec la littérature [9]. Lorsque le cristal contient des défauts, les cinétiques de décroissance de cette lumi-

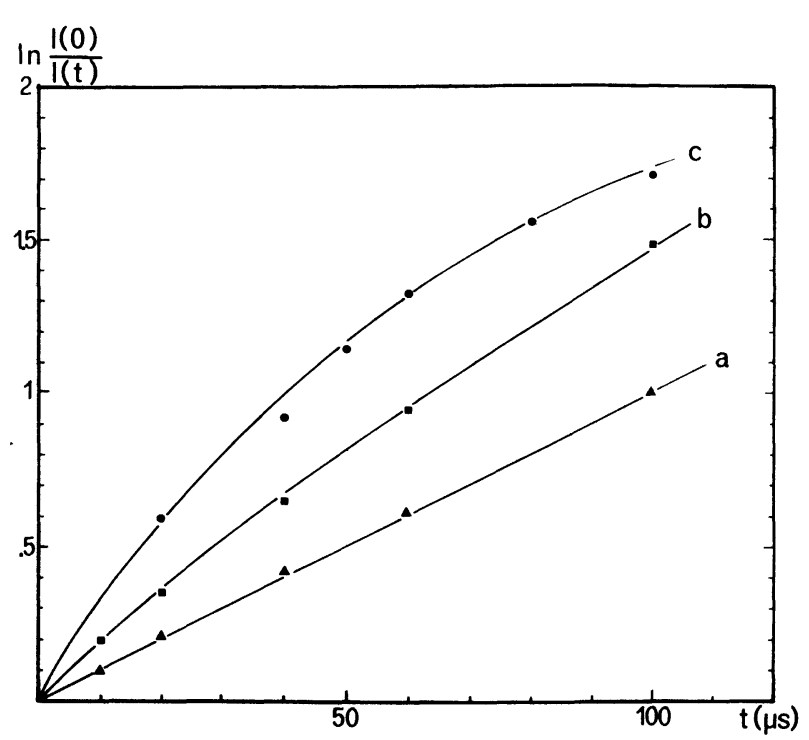

FIG. 3. - Enregistrements de temps de déclin de la luminescence excitonique $I(t) \pi(540 \mathrm{~nm})$ dans $\mathrm{KBr}$ à $10 \mathrm{~K}$ en fonction de la concentration en centres $\mathrm{F}$ formés (Conditions d'irradiation : $30 \mathrm{kV}, 0,5 \mu \mathrm{A} / \mathrm{cm}^{2}$ pulsée à $100 \mathrm{~Hz}$, durée des pulses $0,7 \mathrm{~ms}$ )

\begin{tabular}{ll} 
Concentrations en centres $F$ & $J / J_{0}$ \\
\hline a) 0 & 1 \\
b) $3,9 \times 10^{17} / \mathrm{cm}^{3}$ & 0,214 \\
c) $1,4 \times 10^{18} / \mathrm{cm}^{3}$ & 0,043
\end{tabular}

$J / J_{0}$ est le rapport de l'intensité de la luminescence $\pi$ à $540 \mathrm{~nm}$ d'un cristal irradié en continu à l'intensité $J_{0}$ initiale (cristal vierge). nescence sont plus compliquées ; on peut, néanmoins, prendre comme valeur de $\tau$ le temps nécessaire pour avoir la valeur initiale divisée par $e$. On détermine alors $\tau_{\mathrm{b}}=65 \mu \mathrm{s}$ et $\tau_{\mathrm{c}}=43 \mu \mathrm{s}(\mathrm{a}, \mathrm{b}, \mathrm{c}$ se réfèrent aux indices des enregistrementsde la figure 3 ).

La diminution de l'intensité de la luminescence $\pi$ observée pour les concentrations en centres $F$ par rapport à la valeur du cristal vierge, respectivement 0,214 et 0,043 pour $b$ et $c$, montre alors que le mécanisme d'extinction n'est pas dû à une voie de désexcitation non radiative parallèle à la transition radiative à partir de l'état relaxé excité de l'exciton auto-piégé. Ceci confirme les résultats déjà obtenus sur KI [10]. La variation du temps de déclin de la luminescence, en fonction des défauts introduits, est donc une perturbation du deuxième ordre de l'émission.

Exemple 3 : Cinétiques de croissance des centres $\mathrm{F}$ et $\mathrm{H}$ et de décroissance de la luminescence intrinsèque $\sigma$ de $\mathrm{KBr}$ à $10 \mathrm{~K}$.

Cet exemple illustre la possibilité de comparer deux cinétiques d'absorption optique simultanément en présence d'une forte cathodoluminescence. On enregistre les croissances de deux centres complémentaires formés à $10 \mathrm{~K}$ : le centre $\mathrm{F}$ et le centre $\mathrm{H}$ donnant lieu respectivement aux bandes d'absorption à $601 \mathrm{~nm}$ et $380 \mathrm{~nm}$. En outre, nous avons détecté simultanément l'intensité de la luminescence $\sigma$ (émission à $280 \mathrm{~nm}$ ) due à la recombinaison de l'exciton auto-piégé (montage expérimental de la figure 1).

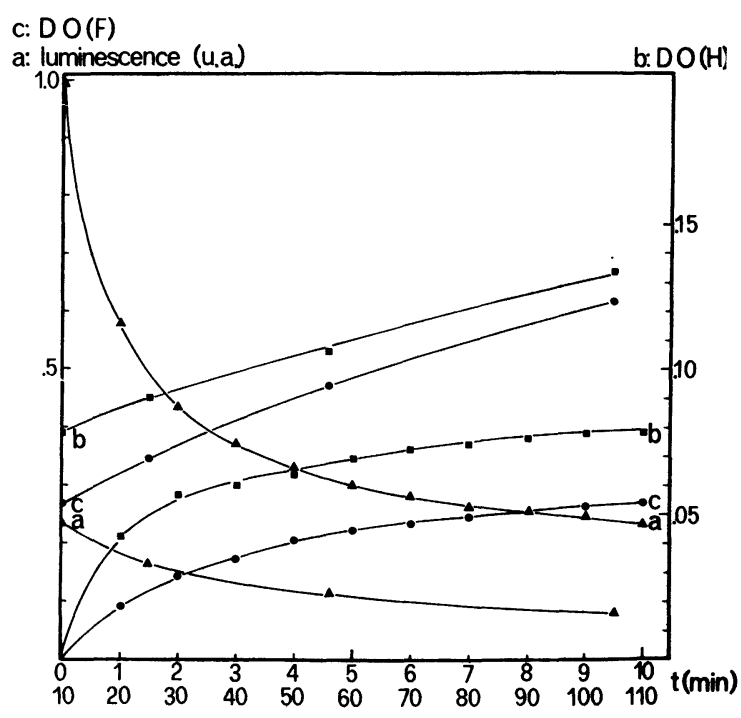

Fig. 4. - Courbe a : cinétique de la luminescence intrinsèque $\sigma$; Courbe $\mathrm{b}$ : cinétique de croissance des centres $\mathrm{H}$; Courbe $\mathrm{c}$ : cinétique de croissance des centres $\mathrm{F}$ dans $\mathrm{KBr}$ à $10 \mathrm{~K}$ (conditions d'irradiation : $30 \mathrm{kV}, 0,5 \mu \mathrm{A} / \mathrm{cm}^{2}$, pulsée à $50 \mathrm{~Hz}$, durée des pulses : $5 \mathrm{~ms}$ ).

La figure 4 montre que la luminescence décroît suivant une loi exponentielle en fonction de la concentration en centres $\mathrm{H}$ :

$$
J=J_{0} \exp (-\beta \mathrm{H}) \quad\left(\beta=6,32 \times 10^{-19} \mathrm{~cm}^{3}\right) .
$$


Nous avons pu ainsi également mettre en évidence le rôle joué par les centres interstitiels dans l'extinction de la luminescence et déterminer le volume $\beta$ d'interaction exciton-centre $\mathbf{H}$.

4. Conclusion. - Notre appareillage permet l'enregistrement simultané d'un certain nombre de paramètres optiques de cristaux sous irradiation électronique ou excitation UV. Il est possible ainsi d'étudier en même temps des propriétés de luminescence (cinétique, analyse spectrale, durée de vie) et de création de défauts (cinétique et spectre d'absorption, enregistrement de deux cinétiques à deux longueurs d'onde différentes et dans des zones d'émission de cathodoluminescence).

Par sa grande souplesse d'emploi (large gamme de taux de déposition d'énergie, fonctionnement en continu ou en pulsé), il est particulièrement bien adapté à l'étude des défauts dans les halogénures alcalins et, d'une manière plus générale, à celle des matériaux dont les propriétés de luminescence se dégradent sous irradiation ionisante.

\section{Bibliographie}

[1] Levy, P. W., Lengweiler, K., Carrera, N. J., Mattern, P. L., International Conference on Color Centers in Ionic Crystals, Sendaï. Abstract H 185 (1974).

[2] LamatsCh, H., Thèse, Université de Neuchâtel (1971).

[3] Herrmann; F., Thèse, Université de Lyon (1971).

[4] Townsend, P. D., J. Phys. C-Solid State Phys. 9 (1976) 1871.

[5] Goldstein, F. T., Phys. Stat. Solids 20 (1967) 379.
[6] Guillot, G., Pinard, P., Cryst. Lat. Def. 5 (1974) 113.

[7] Tanimura, K., Okada, T., Phys. Rev. B 13 (1976) 1811.

[8] Nouailhat, A., Guillot, G., Mercier, E., Solid State Commun. 18 (1976) 399.

[9] Pooley, D., Runciman, W. A., J. Phys. C 3 (1970) 1815.

[10] Guillot, G., Nouailhat, A., Mercier, E., Pinard, P., Int. Conf. Luminescence Tokyo (1975), J. of Luminescence 1213 (1976) 327. 